\title{
Observations of an Unexpected Meteor Shower Outburst at High Ecliptic Southern Latitude and Its Potential Origin
}

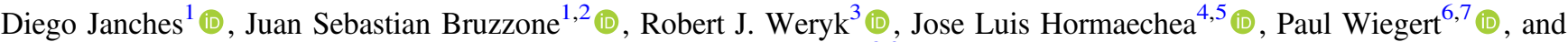 \\ Claudio Brunini ${ }^{8,9}$ \\ ${ }^{1}$ ITM Physics Laboratory,NASA/Goddard Space Flight Center, 8800 Greenbelt Road, Greenbelt, MD 20771, USA; diego.janches@nasa.gov \\ ${ }^{2}$ Department of Physics, Catholic University of America, 620 Michigan Avenue, NE, Washington, DC 20064, USA \\ ${ }^{3}$ Institute for Astronomy, University of Hawaii, 2680 Woodlawn Drive, Honolulu, HI 96822, USA \\ ${ }^{4}$ Facultad de Ciencias Astronomicas y Geofísicas, Universidad Nacional de La Plata, La Plata, Argentina \\ ${ }^{5}$ Estación Astronomica Rio Grande, Rio Grande, Tierra del Fuego, Argentina \\ ${ }^{6}$ Deptartment of Physics and Astronomy, The University of Western Ontario, London, Ontario, Canada \\ ${ }^{7}$ Institute for Earth and Space Exploration (IESX), The University of Western Ontario, London, Ontario, Canada \\ ${ }^{8}$ AGGO, CONICET, Villa Elisa, Pcia. de Buenos Aires, Argentina; claudiobrunini@yahoo.comn \\ ${ }^{9}$ Facultad de Ciencias Astronómicas y Geofísicas, Universidad Nacional de La Plata, La Plata, Pcia. de Buenos Aires, Argentina \\ Received 2020 April 8; revised 2020 April 27; accepted 2020 May 8; published 2020 May 26
}

\begin{abstract}
A strong and unexpected meteor shower outburst was observed by the Southern Argentina Agile MEteor Radar Orbital System (SAAMER-OS) at high southern ecliptic latitude within the South Toroidal region. The outburst, which was active throughout solar longitudes $351^{\circ}$ and $352^{\circ}$, peaked at 09:30 UT on 2020 March 12, has a mean Sun-centered ecliptic radiant of $\lambda-\lambda_{0} \sim 307^{\circ} .5$ and $\beta \sim-77^{\circ} .2$ and a geocentric velocity of $30.7 \mathrm{~km} \mathrm{~s}{ }^{-1}$. Using the $D^{\prime}$ parameter criterion, we find the corresponding orbital elements of the outburst to match well with both the $\beta$ Tucanid and $\delta$ Mensid meteor showers, suggesting these are in fact the same shower. We also find a promising parent candidate in asteroid (248590) $2006 \mathrm{CS}$, a large $(D \sim 2 \mathrm{~km})$ highly inclined $52^{\circ}$ near-Earth object.
\end{abstract}

Unified Astronomy Thesaurus concepts: Meteor showers (1034); Meteors (1041); Radar astronomy (1329)

\section{Introduction}

The majority of the incoming meteoroid flux originating from the Zodiacal Dust Cloud is in the mass range of $10^{-14}$ to $10^{-7} \mathrm{~kg}$, which enters the atmosphere at speeds between 11 and $72 \mathrm{~km} \mathrm{~s}^{-1}$ (Ceplecha et al. 1998). The highly energetic collisions with air molecules cause the meteoroids to heat and ablate, and further collisions release both neutral and ionized atoms from the meteoroid. The phenomena associated with a meteoroid impacting a planetary atmosphere are collectively termed a meteor and, on Earth, are typically observable by an assorted class of ground-based radars (Baggaley 2002; Janches et al. 2003, 2015, 2017; Kero et al. 2019). In particular, all-sky very high frequency (VHF) meteor radars detect mainly specular meteor trails, which are generally semi-stationary plasma columns of lower electron line density left in the wake of the meteoroid's trajectory (Baggaley 2002). These systems are generally dedicated to meteor observations, and over time are capable of collecting large data sets of meteoroid statistics. These cover various conditions, namely, the seasonal and diurnal changes to the Earth's orientation and location in space, as well as to monitor for meteor shower activity, and detect both expected and unexpected outbursts (Campbell-Brown \& Wiegert 2009; Janches et al. 2015).

Over the past 50 years, several meteor radar systems were used for detailed orbital surveys both of the Sporadic Meteor Complex and from shower activity. Examples of these are: (1) the Harvard Radio Meteor Project (HRMP; Brown \& Jones 1995; Taylor \& Elford 1998); (2) the Adelaide radio meteor system (Gartrell 1972; Gartrell \& Elford 1975) in Australia; (3) the Advanced Meteor Orbit Radar (AMOR) operated in Christchurch, New Zealand (Galligan \& Baggaley 2004, 2005); and (4) the Canadian Meteor Orbit Radar (CMOR) in Ontario, Canada (Webster et al. 2004). Of these, only CMOR is currently operational. The HRMP observations provided about $2 \times 10^{4}$ meteoroid orbits from 1968 to 1969 , which have been used for many existing near-Earth meteoroid stream searches (Sekanina 1973) and environment models (Drolshagen et al. 2008). A much larger study, but in the southern hemisphere, was conducted by AMOR, which observed approximately $5 \times 10^{5}$ orbits over the course of five years from 1995 to 1999 (Galligan \& Baggaley 2004, 2005). The still-operational CMOR system has recorded over 15 million individual meteoroid orbits for particles with mean mass near $10^{-7} \mathrm{~kg}$ from 2002 to date and each day $\sim 4000-5000$ orbits are added (Brown et al. 2010).

The continual survey of meteor showers is compelling because it provides information on the cyclic annual strength in the activity of showers, and thus constrains models of dust evolution in the solar system. Furthermore, weaker and/or minor streams require multiyear observations in order to obtain sufficient statistics to be observed over the sporadic background (Brown et al. 2010). More observations, including multiyears, seasons, and sky coverage, enable the identification of expected and/or unexpected outbursts. For example, Jenniskens et al. (2016a) reported a Volantid shower outburst, a high southern ecliptic latitude shower that showed unexpected high activity only during 2015 New Year's Eve according to both optical and radar measurements (Younger et al. 2016; Pokorný et al. 2017). More recently, comparisons between radar and optical observations have shown specific showers to have differences in the evolutionary stages of their different mass ranges (Jeniskens et al. 2018; Bruzzone et al. 2020). Thus, filling the observational gap in the southern hemisphere is of critical importance.

Since 2012, the Southern Argentina Agile MEteor RadarOrbital System (SAAMER-OS), the latest meteor radar conducting continuous meteor orbital observations in the 
southern hemisphere, has filled that gap (Janches et al. 2015). Enabled by several recent software and hardware upgrades, this system currently records over 15,000 daily orbits, an unprecedented high statistic, since 2019 and has collected more than $10 \mathrm{M}$ orbits since it began operations. In this work, we report an unexpected outburst observed by SAAMER-OS during 2020 March 12-13. We describe the radar and our shower search methodology in Section 2, and provide the results and characteristics of the outburst in Section 3. We also attempt to link the outburst to a parent body. Finally, we provide final remarks and conclusions in Section 4.

\section{Instrumentation and Data Analysis}

SAAMER-OS is hosted at the Estacion Astronómica Rio Grande (EARG), located in Rio Grande, Tierra del Fuego, Argentina. It has evolved significantly from its original configuration reported in Janches et al. (2015), currently consisting of five distinct radar stations: the central station (SAAMER-C; $53^{\circ} .786 \mathrm{~S}, 67^{\circ} .751 \mathrm{~W}$ ) that hosts the transmitting and interferometry-enabled receiving antenna arrays; SAAMER-N (53.682 S, 67.871 W) located approximately $13 \mathrm{~km}$ northwest of the central station; SAAMER-W (53:828 S, $\left.67^{\circ} .842 \mathrm{~W}\right)$ located approximately $7 \mathrm{~km}$ southwest of the central station; SAAMER-S $\left(53^{\circ} .852 \mathrm{~S}, 67^{\circ} .76 \mathrm{~W}\right)$ located approximately $7 \mathrm{~km}$ south of the central station; and SAAMER-E (53.772 S, 67.727 W) located approximately $4 \mathrm{~km}$ northeast of the central station. Each of the four noncentral remote sites host a single three-element crossed yagis receiving antenna identical to those forming the interferometer array at SAAMER-C to detect the forward scatter signal from a meteor trail. The radar transmits at $32.55 \mathrm{MHz}$ with a peak power of 64 $\mathrm{kW}$, and currently uses a pulse repetition frequency of $625 \mathrm{~Hz}$.

The original design of SAAMER-OS' transmitter (TX) configuration was optimized to perform gravity wave studies. This required the detection of meteors between zenith angles of $15^{\circ}$ and $55^{\circ}$ (Fritts et al. 2010a, 2010b). For that purpose, eight three-element crossed yagis were deployed arranged in a circle of diameter $27.6 \mathrm{~m}$. Each transmitting in opposite phasing of every other yagi (Janches et al. 2014). In 2019, the system transmission strategy was upgraded with the deployment of a single new TX antenna with the goal of improving the detection rate of meteors at larger zenith angles. (Fritts et al. 2010a, 2010b; Janches et al. 2014). By concentrating the full power of SAAMER in one TX antenna, a more uniform detection pattern is achieved that satisfies this original requirement, but also increases the number of events detected at larger zenith angles.

A second critical upgrade entailed the optimization of the receive signal path of the existing remote receiving stations. Enhancements include the installation of low noise amplifiers at the receive antennas and upgrades of the receiving and data acquisition hardware for improved signal filtering and selectivity and to increase dynamic range, which improve the ability of the radar to detect weaker meteor echoes. In addition, modification of the data processing software was also implemented in order to recognize "overdense" meteors (produced by larger and more energetic meteoroid particles), which also improves meteor detection rates. Before this modification, the radar only recorded the lower-returned power "underdense" meteor echoes, which are used to estimate mesospheric wind speeds (Fritts et al. 2010b; Janches et al. 2019). The software upgrade was achieved by implementing detection and orbital determination software that runs parallel to the standard SKiYMET meteor radar systems' software (SKICORR; Hocking et al. 2001). Specifically, echoes are detected in a multistage process. After determining the appropriate noise thresholds for a given streamed "dump. raw" file, the first stage identifies candidate echoes that exceed the background noise level by some set level (for SAAMER$\mathrm{OS}$, the limit is $6.0 \sigma$ ). Once all echoes are found, they are analyzed by a second stage that determines the interferometry solution, the decay constant, the time inflection picks (corresponding to when the meteoroid reaches the specular point; see McKinley 1961), and then a record of each echo is saved to disk. The interferometry solution, determined from the five-receiver channel typical of these systems (Hocking et al. 2001), defines a plane that must contain the meteor trail. As well, the inflection points from all available remote receiving stations (minimum of three, including the central transmitting site) are used to define a vertical plane that also contains the echo (Jones et al. 2005). The intersection of these two planes defines the meteor trajectory, which allows for a direct measure of its velocity vector that is then transformed into Keplerian orbital elements. The software that does event detection, correlation, and time of flight calculation was first used on CMOR (Weryk \& Brown 2012) but has been modified to produce orbits incrementally throughout the day so shower outbursts can be found on more immediate timescales.

To search for and identify showers, we employ a 3D wavelet transform to isolate and characterize our daily detections. This method was first applied by AMOR to identify shower structure in radar data (Baggaley et al. 1994; Galligan \& Baggaley 2002), but has since also been applied to other radar meteor surveys (Brown et al. 2008, 2010; Pokorný et al. 2017; Schult et al. 2018) as well as a more recent video survey (Bruzzone et al. 2020). The wavelet transform is well suited to isolate radiant enhancements at various scales in the radiant coordinates and time. Meteors belonging to a specific shower naturally cluster in the radiant coordinate-speed and time domain over a characteristic scale: spread in radiant coordinates, speed, and activity period. Such a grouping of radiants contrasts with the large-scale radiant distribution of the sparse sporadic background. Therefore, a given meteor radiant distribution can be probed with the wavelet transform to reveal enhancements at different scales. As with previous radar studies, we employ the 3D Mexican hat wavelet transform over a radiant distribution and geocentric speed (Brown et al. 2010). The width of the kernel can be adjusted accordingly to resemble the true spread in angular coordinates and speed of the radiant distribution.

The computation of the wavelet is achieved in geocentric Sun-centered ecliptic coordinates: $\lambda-\lambda_{0}$ and $\beta$, in degrees, and geocentric speed $\left(V_{g}\right)$ in $\mathrm{km} \mathrm{s}^{-1}$ and binned by one degree in solar longitude $\left(\lambda_{0}\right)$. By choosing this reference frame, we remove the natural motion of the Sun while minimizing the radiant drift with time. For our daily shower search, the wavelet is evaluated at 0.50 steps in spatial coordinates and $5 \%$ steps in $V_{g}$ while advancing at $1^{\circ}$ steps in $\lambda_{0}$. The procedure returns a list of wavelet coefficients from which a yearly median and standard deviation are computed, and a $3 \sigma$ rejection is applied to remove outliers. For those wavelet coefficients greater than $3 \sigma$, the maximum is stored and a list of wavelet maxima created. We proceed to identify a shower core radiant candidate as the radiant producing the wavelet coefficient global maxima. 

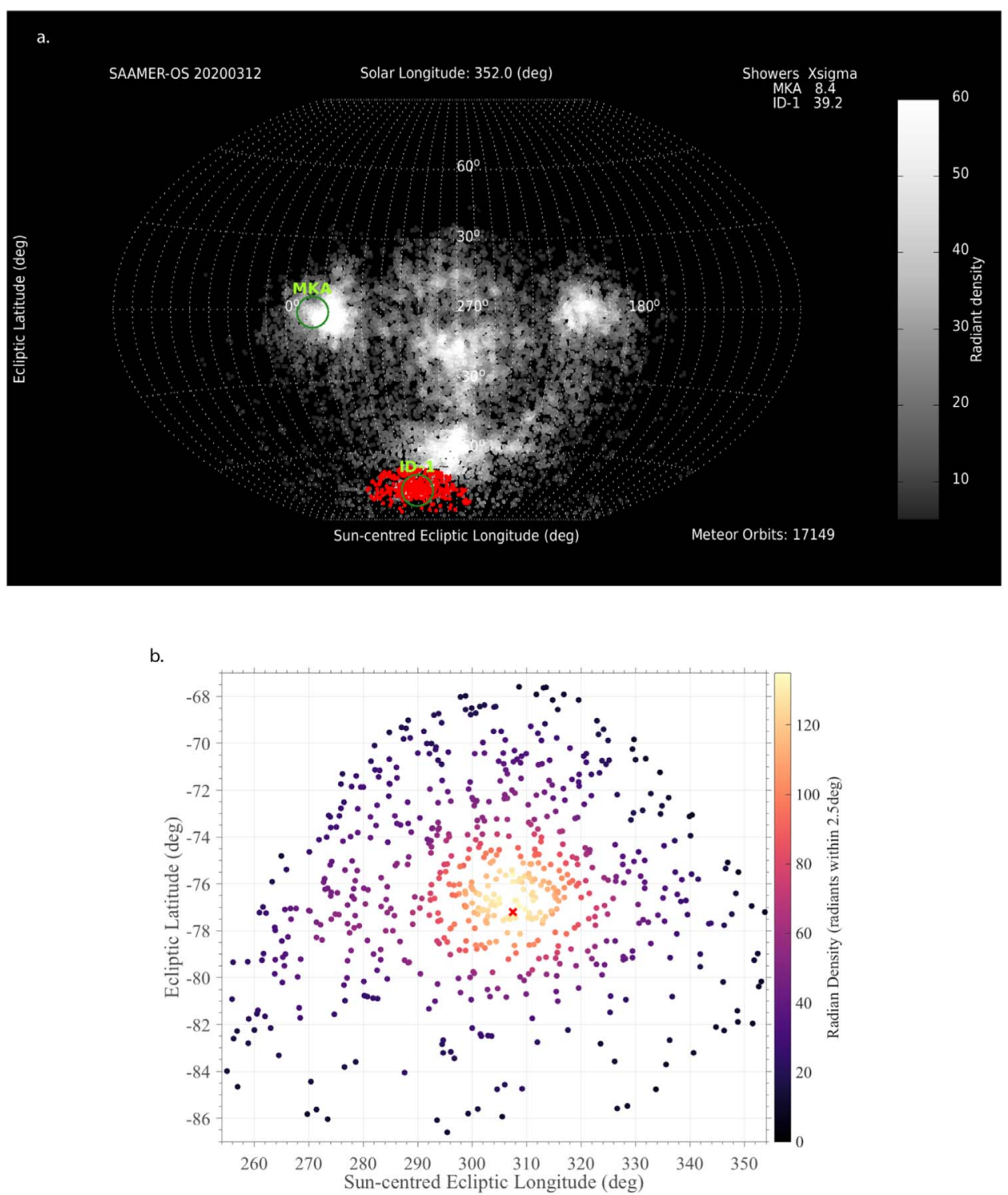

Figure 1. (a) Sun-centered ecliptic map displaying all the meteors detected on 2020 March 12 (white dots) with meteors belonging to the outburst superimposed (red dots). The outburst is identified at ID-1. The wavelet also identified the presence of the Daytime kappa Aquariids (IAU\#128 MKA). (b) Sun-centered meteoroid radiants detected by SAAMER-OS on March 12-13 color coded by radiant density. The red cross indicates the center of the shower at $\lambda-\lambda_{0}=307.5$ and $\beta=-77^{\circ} .2$.

With the candidate shower core location identified, we cross reference it against a published list of meteor showers (Brown et al. 2010; Pokorný et al. 2017). When the location of the candidate shower is within $3^{\circ}$ and $15 \%$ in geocentric speed of a known shower, the radiant position is labeled with the shower IAU code in the radiant map. Currently, potential outburst or unknown shower identification is performed by visually examining the daily reports resulting from this procedure; however, we are currently automating this step to run in near realtime. For more details of the analysis procedure the reader can refer to Bruzzone et al. (2020). For this study, however, we revisit our daily report with the detection of the outburst but repeat the wavelet transform technique at a much finer resolution in angular and velocity space at 0.1 and $1.5 \%$, respectively, thus allowing for more precise radiant position and speed determination.

\section{Results and Discussion}

Figure 1(a) shows the ecliptic sky in Sun-centered ecliptic coordinates where the white dots represent all the meteor radiants detected during a $24 \mathrm{hr}$ period on 2020 March 12, while the red dots show those meteors identified as part of the shower during the entire period of the outburst. Figure 1(b) shows the radiants of the 541 meteors identified as those belonging to the outburst, color coded by radiant density within a $\sim 10^{\circ} \times 10^{\circ}$ window around the center of the shower as estimated by the wavelet analysis. To identify the meteors associated with the outburst, we use the wavelet-based estimation of the outburst radiant position and speed in addition to a radiant density map as a visual guide to help with the identification procedure. We extract meteors within $10^{\circ}$ of the geocentric radiant position and $15 \%$ of speed corresponding to the wavelet maximum as our sample of the outburst. Each event had the time picks for all stations manually verified, and were mostly observed between 


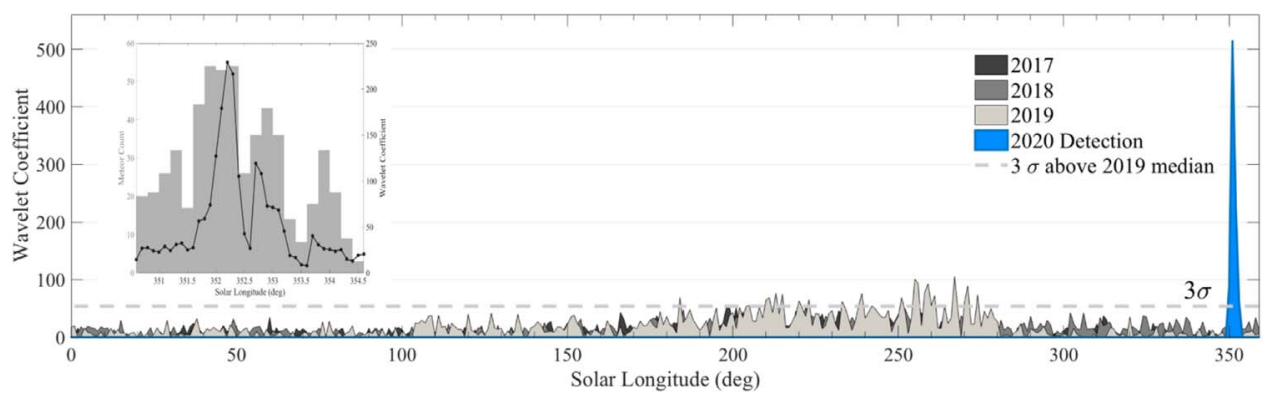

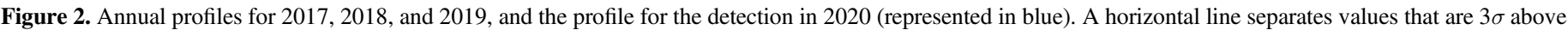

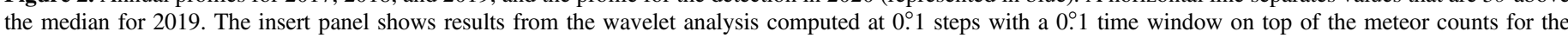
shower meteors (shaded histogram).

$\lambda_{0} \sim 350^{\circ} .5$ and $353^{\circ} .0$ (see insert in Figure 2). We recognize that there may be some sporadic contamination, but given the strength of this outburst, this is minimal ( $\sim 30$ meteors total based on previous years within same extraction radius). Moreover, we set the wavelet kernel to enhance scales in phase space typical of showers, which are much smaller than the width of the sporadic sources and therefore not sensitive to the sporadic background (Bruzzone et al. 2015). Figure 2 displays the annual activity profiles represented by the wavelet coefficient $\left(W_{c}\right.$; Bruzzone et al. 2020) for meteors within a $\sim 1^{\circ} \times 1^{\circ}$ box centered around the radiant position of the outburst as estimated by the wavelet analysis for 2017, 2018, and 2019 (gray shades), and the profile for the detection in 2020 (represented by the blue shaded area). The horizontal line in Figure 2 represents the $3 \sigma$ threshold above the median estimated in 2019. The insert in Figure 2 displays the wavelet coefficients (black line, right vertical axis) computed at 0.1 steps for $\left(\lambda-\lambda_{0}, \beta\right)$ with a 0.1 in time (i.e., $\left.\lambda_{0}\right)$. The line is displayed on top of the meteor counts determined by our filtering methodology (shaded histogram, left vertical axis). Fitting a Gaussian curve to the histogram indicates a peak of the outburst occurring at $\lambda_{0} \sim 352^{\circ} .01$ with a peak uncertainty equal to 0.1 corresponding to 9:30UT on March 12 2020. The peak of the histogram includes 113 meteors with a determined maximum $W_{c}$, i.e., center of the outburst, at $\lambda-\lambda_{0} \sim 307^{\circ} .5$ and $\beta \sim-77^{\circ} .2$ (geocentric R.A. and decl. of $\alpha=62^{\circ} .5$, $\delta=-76^{\circ} .2$, respectively) and a geocentric speed, corrected for deceleration (Bruzzone et al. 2020), of $V_{g} \sim 30.3 \mathrm{~km} \mathrm{~s}^{-1}$. It is important to note the dips in the histogram that are most likely due to the detectability of the shower varying throughout the day due to the changing geometry of the outburst radiant with respect to the radar beam. A proper location of the peak will require a correction of this curve taking into account the radar effective collecting area, which will be done in future work, but we do not expect to change significantly.

As in previous radar studies (Brown et al. 2010; Schult et al. 2018; Pokorný et al. 2019; Bruzzone et al. 2020), we repeat the wavelet-based procedure at $1^{\circ}$ steps in $\lambda_{0}$ including meteors within $1^{\circ}$ at each step in order to improve our sample and include most of the observed activity period. The wavelet technique returns a peak $W_{c}$ at $\lambda_{0}=352^{\circ} .0$, geocentric $\lambda-\lambda_{0}=305^{\circ} .73$ and $\beta=-77^{\circ} .2 \quad\left(\alpha=63^{\circ} .6 \quad\right.$ and $\delta=-76^{\circ} .6$ ), and $V_{g}=30.69 \mathrm{~km} \mathrm{~s}^{-1}$ employing 390 meteors. Given the larger sample, we therefore use this estimate of the shower radiant position and speed to derive the nominal orbital elements listed in Table 1.

Meteor showers in this region of the sky and during this time period are scarce. Gartrell \& Elford (1975) reported the discovery of the $\beta$ Tucanid shower (IAU\#108; BTU), identified as two separate showers containing a handful of meteors at $\alpha=51^{\circ}, \delta=-81^{\circ}$ and $\alpha=50^{\circ}, \delta=-78^{\circ}$. However, Kronk (2014) associated this region with the $\delta$ Mensid shower (IAU\#130, DME), later confirmed by Jenniskens et al. (2016b) utilizing video observations with the New Zealand Cameras for All-sky Meteor Surveillance (CAMS) station. The authors reported 11 meteors recorded with CAMS between $\lambda_{0} \sim 357^{\circ}$ and $2^{\circ}$ (March 17-22) with a mean velocity somewhat larger than our observations $\left(V_{g}=34.4 \mathrm{~km} \mathrm{~s}^{-1}\right)$. Furthermore, Gartrell (1972) and Gartrell \& Elford (1975) associated the BTU shower with comet C/ 1976 D1 (Bradfield), while Jenniskens et al. (2016b) associate the DME with comet C/1804 E1 (Pons). According to Kronk (2014), no newer records appear to exist for either the DME or BTU showers after the report by Gartrell \& Elford (1975) (not counting the occasional 1-2 meteors per hours in the 1970s and 1980s by observers in the southern hemisphere) until the DME confirmation by Jenniskens et al. (2016b). Certainly, no outburst from this region was ever reported.

The SAAMER-OS-observed outburst occurred during a period in between that of the BTU (February 27-March 2; Jenniskens 2006) and the DME (Jenniskens et al. 2016b). The mean Sun-centered ecliptic longitude of the DME meteor cluster reported by Jenniskens et al. (2016b) appears to be significantly westward of that reported by SAAMER-OS. Observations with SAAMER-OS previous to 2020 show indications that the shower was present during the same time, but with $W_{c}$ values just above the $3 \sigma$ threshold. Also, the SAAMER-OS identification procedure follows potential showers and classifies them as such when the enhancements in the wavelet analysis are present for at least three days. This shower appears to last only two days in the radar observations and thus was never singled out until the 2020 outburst.

We performed an attempt to associate the outburst with known showers and potential parent bodies using the $D^{\prime}$ criterion (Drummond 1981). The results are also listed in Table 1. Uncertainties in nominal orbital elements are drawn from a Monte Carlo procedure based on the errors in shower radiant position and speed. Following Bruzzone et al. (2020), the error in shower radiant position is estimated as the angular difference of the wavelet radiant position and the position of the observed peak in radiant density. For the error in shower speed, we adopt the standard error of the mean shower geocentric speed. Although the time of occurrence is between the recorded observation times of both the BTU and DME showers, the observed outburst matches well to both showers suggesting that these are in fact the same shower in agreement 
Table 1

Orbital Elements for the Outburst and Parent Candidates

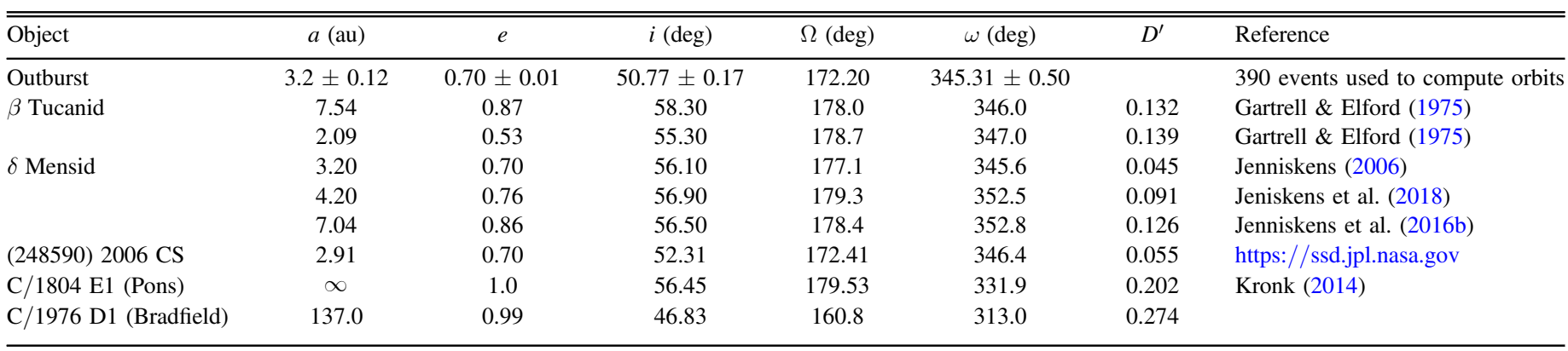

Note. Uncertainties in nominal orbital elements for the outburst are drawn from $10^{4}$ Monte Carlo iterations using errors in shower radiant position and speed of 0.36 and $0.1 \mathrm{~km} \mathrm{~s}^{-1}$, respectively.

with Jenniskens et al. (2016b). The mean $D^{\prime}$ between the shower peak and the various BTU orbital elements determinations is $\sim 0.14$ and DME is slightly better $\sim 0.09$. Our analysis also suggests that asteroid (248590) $2006 \mathrm{CS}$, a $\sim 2 \mathrm{~km}$ highly inclined $\left(i \sim 52^{\circ}\right)$ near-Earth object (NEO), is a better candidate than C/1976 D1 (Bradfield) proposed by Gartrell \& Elford (1975) for the BTU, which has a $D^{\prime} \sim 0.3$, or C/1804 E1 (Pons) proposed by Kronk (2014) and Jenniskens et al. (2016b) for the DME $\left(D^{\prime} \sim 0.2\right.$; Table 1$)$. The $D^{\prime}$ between the peak of the shower and the asteroid is 0.055 , with a variability of 0.001 within the determined outburst orbital parameters uncertainty. The next best match in our analysis is Asteroid $2016 \mathrm{DK}$ with a $D^{\prime}$ equal to 0.169 . Furthermore, there is only a 1 in 1200 chance of this being a coincident match. This probability is computed by a Monte Carlo process, where hypothetical NEOs are drawn at random from the theoretical NEO distribution of Greenstreet et al. (2012) until a selection is made with a smaller $D^{\prime}$ than the one in question (in this case, $\left.D^{\prime}=0.055\right)$. Over 10 trials, an average of $4.0 \times 10^{5}$ draws were required before a better match was obtained. Asteroid $2006 \mathrm{CS}$ has an absolute magnitude $H=16.4$ (corresponding to a diameter of $2.3 \mathrm{~km}$ at an albedo of 0.09 ), and there are only 330 NEOs of this size or larger in near-Earth space according to Mainzer et al. (2011). Thus, there is only a $330 / 4 \times 10^{5} \approx 1 / 1200$ probability that the small $D^{\prime}$ value between the shower and asteroid occurred by chance. Although this does not guarantee this asteroid is the parent, it does reveal that is extremely unlikely that a sizable undiscovered NEO will be found with a more similar orbit.

\section{Conclusions}

In this Letter, we reported the observations of an unexpected very active meteor shower outburst utilizing the Southern Argentina Agile MEteor Radar Orbital System (SAAMEROS). The event was located at a high southern ecliptic latitude $\left(\lambda-\lambda_{0} \sim 307^{\circ} .5\right.$ and $\beta \sim-77^{\circ} .2$ ) within the South Toroidal region and lasted approximately 2 days $\left(351^{\circ} \leqslant \lambda_{0} \leqslant 353^{\circ}\right)$. The peak of the activity was determined to occur at 09:30 UT on 2020 March $12\left(\lambda_{0} \approx 352.0^{\circ}\right)$ at a geocentric velocity (corrected for deceleration and zenith attraction) of $30.7 \mathrm{~km} \mathrm{~s}^{-1}$. Using the $D^{\prime}$ parameter criterion, we find the nominal orbital characteristics of the outburst to match well with both the $\beta$ Tucanid and the $\delta$ Mensid meteor showers, indicating these are likely the same shower as previously suggested by Jenniskens et al. (2016b). The period of observation of the outburst is between those reported previously for the BTU and DME showers (February 27March 2 and March 16-22, respectively; Jenniskens et al. 2016 b), but the mean velocity seems to be $\sim 4 \mathrm{~km} \mathrm{~s}^{-1}$ slower than previously reported. Since radar observations observe smaller particles than video techniques, and because SAAMER-OS is a far more sensitive instrument than the radar used by Gartrell \& Elford (1975), we expect the outburst to be composed of smaller particles than previously observed $(m \sim 1$ to $100 \mu \mathrm{g}$ ). Thus, the observed differences suggest that the outburst may have had significantly different evolutionary characteristics than what was observed in previous reports, but we note that a full analysis of the echo power profiles must be performed to help characterize the mass index of the shower. This is required for a long-term orbital evolution to identify when exactly the stream was created. In order to do this, however, it requires the receivers to be power calibrated first. This calibration is currently being planned to take place late in 2020.

We found that asteroid (248590) 2006 CS is a promising parent candidate. This is a large-sized NEO with a diameter $\mathrm{D} \sim 2 \mathrm{~km}$ and a highly inclined orbit with inclination $i \sim 52^{\circ}$. While it does not have any reports of cometary activity, its heliocentric orbit is much like that of a Jupiter-family comet (Tisserand's constant with respect to Jupiter is 2.44), and it has been recognized previously as a potential dormant comet (Kim et al. 2014). In fact, Jenniskens (2008) suggested this to be the potential parent body of the DME shower, before later proposing comet $\mathrm{C} / 1804 \mathrm{E} 1$ (Pons) as the true parent body (Jenniskens et al. 2016b), even though E1 has a (presumed) parabolic orbit, having left the inner solar system long ago. It is difficult to understand how any release meteoroids would reach the Earth only now, having been ejected long ago when the comet was a large heliocentric distances. Thus, if Pons were really on a rather long period orbit, it probably could not be the source of the outburst. Figure 3 shows (with a reference to the animated form) the orbits of the outburst and various showers and potential parent bodies discussed in this work.

At the time of this report, Asteroid 2006 CS was located more than 3 au from the Sun, approaching perihelion, in the daytime sky and it will not be in the night sky, optimal for optical observations, until the summer of 2020. Inspecting images from the Pan-STARRS (Chambers et al. 2016) image archive, this object does not show any apparent cometary activity, including when it appeared the brightest on 2016 July 16 when it was 1.46 au from the Sun. If 2006 CS is in fact the parent body, it may be an inactive or defunct comet, with the outburst composed of much older material. An alternative 


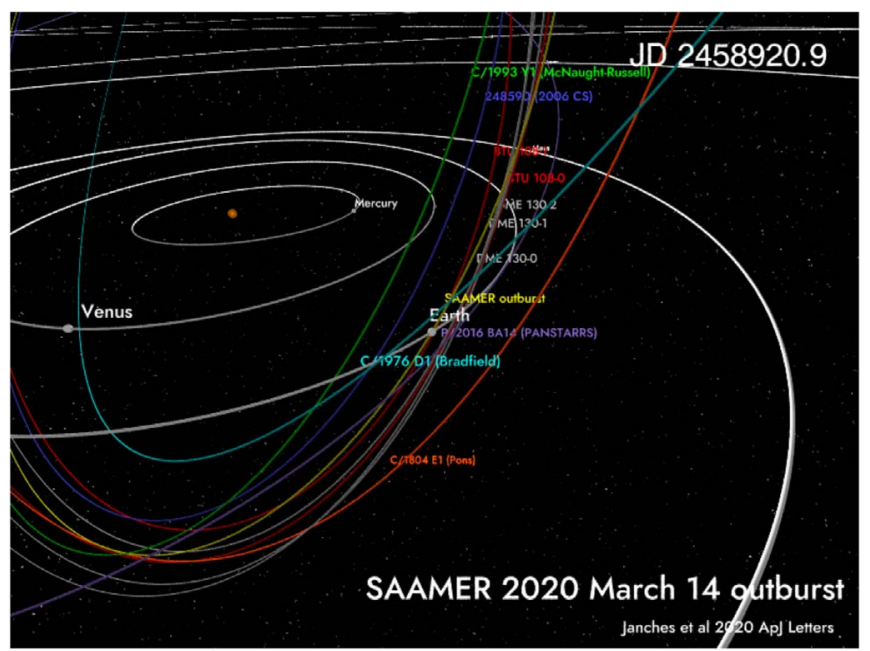

Figure 3. A view of the orbits discussed in this work. Shower orbits included are BTU-108-0 and BTU-108-1, two orbits for the $\beta$ Tucanids from Gartrell \& Elford (1975); as well as three orbits for the $\delta$ Mensids: DME-130-0 (Jenniskens 2006), DME-130-1 (Jeniskens et al. 2018), and DME-130-2 (Jenniskens et al. 2016a). (For review, the animation is at http://www.astro. uwo.ca/ wiegert/SAAMER/SAAMER-outburst-14Mar2020.mp4.)

stream formation theory is that it was somehow produced collisionally, potentially meaning much larger fragments could still exist in space.

D.J. and J.S.B.'s work is supported by the NASA SSO Program and the NASA NESC. R.J.W. was supported through NASA grant 80NSSC18K0656. P.W. received support from the Natural Sciences and Engineering Research Council of Canada (grant No. RGPIN-2018-05659). SAAMER's operation is supported by NASA SSO, NESC assessment TI-1701204, and NSF grant AGS-1647354. The authors appreciate the invaluable support of Carlos Ferrer, Gerardo Connon, and Luis Barbero with the operation of SAAMER-OS.

\section{ORCID iDs}

Diego Janches (iD https://orcid.org/0000-0001-8615-5166 Juan Sebastian Bruzzone (iD https://orcid.org/0000-00022731-0397

Robert J. Weryk (iD https://orcid.org/0000-0002-0439-9341

Jose Luis Hormaechea (i) https://orcid.org/0000-00034533-3282

Paul Wiegert (ib https://orcid.org/0000-0002-1914-5352

\section{References}

Baggaley, W. 2002, in Meteors in the Earth's Atmosphere, ed. E. Murad \& I. Williams (Cambridge: Cambridge Univ. Press), 123
Baggaley, W., Bennett, R., Steel, D., \& Taylor, A. 1994, QJRAS, 35, 293 Brown, P., \& Jones, J. 1995, EM\&P, 68, 223

Brown, P., Weryk, R. J., Wong, D. K., \& Jones, J. 2008, EM\&P, 102, 209 Brown, P., Wong, D. K., Weryk, R. J., \& Wiegert, P. 2010, Icar, 207, 66

Bruzzone, J., Janches, D., Jenniskens, P., Weryk, R., \& Hormaechea, J. 2020, P\&SS, 188, 104936

Bruzzone, J. S., Brown, P., Weryk, R. J., \& Campbell-Brown, M. D. 2015, MNRAS, 446, 1625

Campbell-Brown, M., \& Wiegert, P. 2009, M\&PS, 44, 1837

Ceplecha, Z., Borovička, J., Elford, W., et al. 1998, SSRv, 84, 327

Chambers, K. C., Magnier, E. A., Metcalfe, N., et al. 2016, arXiv:1612.05560

Drolshagen, G., Dikarev, V., Landgraf, M., Krag, H., \& Kuiper, W. 2008, EM\&P, 102, 191

Drummond, J. D. 1981, Icar, 45, 545

Fritts, D. C., Janches, D., \& Hocking, W. K. 2010a, JGRD, 115, 19123

Fritts, D. C., Janches, D., Iimura, H., et al. 2010b, JGRD, 115, 18112

Galligan, D. P., \& Baggaley, W. J. 2002, in IAU Coll. 181, Dust in the Solar System and Other Planetary Systems, ed. S. F. Green et al. (Oxford: Pergamon), 48

Galligan, D. P., \& Baggaley, W. J. 2004, MNRAS, 353, 422

Galligan, D. P., \& Baggaley, W. J. 2005, MNRAS, 359, 551

Gartrell, G. 1972, PASA, 2, 89

Gartrell, G., \& Elford, W. G. 1975, AuJPh, 28, 591

Greenstreet, S., Ngo, H., \& Gladman, B. 2012, Icar, 217, 355

Hocking, W. K., Fuller, B., \& Vandepeer, B. 2001, JASTP, 63, 155

Janches, D., Brunini, C., \& Hormaechea, J. L. 2019, AJ, 157, 240

Janches, D., Close, S., Hormaechea, J. L., et al. 2015, ApJ, 809, 36

Janches, D., Hocking, W., Pifko, S., et al. 2014, JGRA, 119, 2269

Janches, D., Nolan, M., Meisel, D., et al. 2003, JGRA, 108, 1222

Janches, D., Swarnalingam, N., Carrillo-Sanchez, J., et al. 2017, ApJ, 843, 1

Jeniskens, P., Baggaley, J., Crumpton, I., et al. 2018, P\&SS, 154, 21

Jenniskens, P. 2006, Meteor Showers and their Parent Comets (Cambridge: Cambridge Univ. Press)

Jenniskens, P. 2008, Icar, 194, 13

Jenniskens, P., Baggaley, J., Crumpton, I., et al. 2016a, JIMO, 44, 35

Jenniskens, P., Baggaley, J., Crumpton, I., \& Aldous, P. 2016b, JIMO, 44, 187

Jones, J., Brown, P., Ellis, K. J., et al. 2005, P\&SS, 53, 413

Kero, J., Campbell-Brown, M. D., Stober, G., et al. 2019, in Radar Observations of Meteors, ed. G. O. Ryabova, D. J. Asher, \& M. J. Campbell-Brown (Cambridge: Cambridge Univ. Press), 65

Kim, Y., Ishiguro, M., \& Usui, F. 2014, ApJ, 789, 151

Kronk, G. W. 2014, Meteor Showers (Berlin: Springer)

Mainzer, A., Grav, T., Bauer, J., et al. 2011, ApJ, 743, 156

McKinley, D. W. R. 1961, Meteor Science and Engineering (New York: McGraw Hill), 1

Pokorný, P., Janches, D., Brown, P. G., \& Hormaechea, J. L. 2017, Icar, 290,162

Pokorný, P., Janches, D., Sarantos, M., et al. 2019, JGRE, 124, 752

Schult, C., Brown, P., Pokorný, P., Stober, G., \& Chau, J. L. 2018, Icar, 309,177

Sekanina, Z. 1973, Icar, 18, 253

Taylor, A., \& Elford, W. 1998, EP\&S, 50, 569

Webster, A. R., Brown, P. G., Jones, J., Ellis, K. J., \& Campbell-Brown, M. 2004, ACPD, 4, 1181

Weryk, R. J., \& Brown, P. G. 2012, P\&SS, 62, 132

Younger, J., Reid, I., \& Murphy, D. 2016, in Proc. International Meteor Conference, ed. A. Roggemans \& P. Roggemans (Hove, BE: IMO), 352 